\title{
Psychological Symptoms Among Patients With BCR-ABL-Negative Myeloproliferative Neoplasms
}

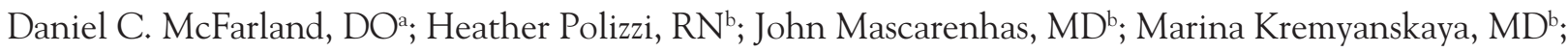 \\ Jimmie Holland, MD; ; and Ronald Hoffman, $\mathrm{MD}^{\mathrm{b}}$
}

\begin{abstract}
Background: BCR-ABL-negative myeloproliferative neoplasms (MPNs) represent a heterogeneous group of diseases, including essential thrombocythemia (ET), polycythemia vera (PV), and myelofibrosis (MF). Psychological manifestations among these diseases have not been adequately described. Methods: Cross-sectional surveys measuring distress, anxiety, and depression were collected from patients with BCRABL-negative MPNs from May 2015 to October 2015. Participants provided demographic information and completed the Distress Thermometer and Problem List (DT\&PL) to assess distress and the Hospital Anxiety and Depression Scale (HADS) to assess distress, anxiety, and depression. They provided information on how their MPN affected their lives. Results: Of the 117 participants, 31.2\% had PV, 28.4\% had ET, $28.4 \%$ had MF, and $11.9 \%$ had another type of MPN. Time with MPN varied from less than 1 year $(7.5 \%), 1$ to 3 years (19.8\%), 3 to 5 years $(23.6 \%), 5$ to 10 years (19.8\%), and more than 10 years (29.2\%). Distress averaged 3.14 (SD, 2.83; DT\&PL), with $40.4 \%$ meeting NCCN criteria for distress, and averaged 8.97 (SD, 7.44; HADS), with 38.5\% meeting HADS criteria for distress. Anxiety averaged 5.54 (SD, 4.37 ), with $31.3 \%$ meeting HADS criteria for anxiety. Depression averaged 3.4 (SD, 3.4), with $12.5 \%$ meeting HADS criteria for depression. Distress was higher for PV (3.86), MF (3.12), and "other" MPN (4.33) than it was for ET (1.81; $P=.016)$. Distress was more common in non-white patients $(P=.015)$ and those with either PV or MF but not ET (DT\&PL $\geq 4 ; P=.038$ ). Patients' comments described coping strategies or symptom burden. Conclusions: Distress and anxiety are highly prevalent with BCR-ABL-negative MPNs and may correspond to disease-related symptom burden. These findings deserve further study.
\end{abstract}

J Natl Compr Canc Netw 2016;14(12):1563-1570

\section{Background}

The prevalence of distress, anxiety, and depressive symptoms has not been assessed by standard measures in patients with BCR-ABL-negative myeloproliferative neoplasms (MPNs). This is perhaps surprising given the large symptom burden that is frequently encountered in patients with MPNs over the course of many years. In many malignancies and advanced cancers, symptom burden tends to correlate with the prevalence of distress, anxiety, and depression. ${ }^{1,2}$ In fact, the severity of MPN symptom burden may be equal to or greater than

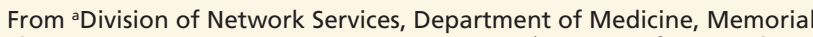
Sloan Kettering Cancer Center, West Harrison; 'Division of Hematology/ Oncology, Tisch Cancer Institute, Icahn School of Medicine at Mount Sinai Hospital, New York; and 'Department of Psychiatry and Behaviora Sciences, Memorial Sloan Kettering Cancer Center, New York, New York. Submitted July 18, 2016; accepted for publication August 15, 2016. that of patients with metastatic cancer or acute myeloid leukemia (AML), in which the prevalence of psychological consequences of cancers has been well studied. ${ }^{3,4}$ In 2007, the first comprehensive assessment of MPN related symptom burden was captured using the Brief Fatigue Inventory (BFI) and the Functional Assessment of Cancer Therapy-Anemia (FACT-An). The most common symptoms were significant fatigue $(81 \%)$, pruritus $(53 \%)$, night sweats $(49 \%)$, bone pain $(43 \%)$, fevers $(13 \%)$, weight loss $(10 \%)$, and splenic pain $(4 \%)$. MPN-associated symptom burden and inability to per-

The authors have disclosed that they have no financial interests, arrangements, affiliations, or commercial interests with the manufacturers of any products discussed in this article or their competitors.

Correspondence: Daniel C. McFarland, DO, Memorial Sloan Kettering Cancer Center, 500 Westchester Avenue, West Harrison, NY 10604. E-mail: danielcurtismcfarland@gmail.com 
form activities of daily living (ADLs) were associated with worsening cytopenias and splenomegaly. ${ }^{5}$ However, distress, anxiety, and depression were not analyzed directly using validated tools.

Patients with BCR-ABL-negative MPNs display clinically significant symptom burdens that are similar in severity to the experience of patients with metastatic cancer or AML, but with a much longer period of overall survival. ${ }^{6}$ In addition, their symptom profiles correspond to elevated inflammatory biomarkers, such as tumor necrosis factor (TNF)- $\alpha$, interleukin (IL)-6, and IL-2 and its soluble receptor. ${ }^{7}$ Many of these same proinflammatory cytokines are also elevated in the setting of depression and contribute to the cytokine hypothesis of depression that has been proposed based on "sickness behavior" models that can be induced with the administration of interferon- $\alpha$ therapy. ${ }^{8-11}$ Psychological symptoms associated with MPNs have not been characterized according to their elevations in inflammatory biomarkers and could potentially contribute to additional knowledge concerning the cytokine hypothesis and the underlying biological bases of depression and other psychological states.

The BCR-ABL-negative MPNs represent a heterogeneous group of 3 primary diseases: essential thrombocythemia (ET), polycythemia vera (PV), and myelofibrosis (MF). ${ }^{12}$ Phenotypically, they are characterized by hematopoietic progenitor cell dysplasia and deregulation based on molecular findings (eg, JAK2, CAL-R mutations). ${ }^{13}$ Both PV and ET display distinct patterns of clonal proliferation of myeloid and erythroid cells, which may evolve into MF. Although they all carry a risk of transformation to leukemia, their symptom profiles and disease trajectories vary considerably. Some of their symptom profiles have acquired notoriety based on their pathognomonic features, such as erythromelalgia and aquagenic pruritus. Thus, MPNs constitute a spectrum of considerable and unique symptoms that patients live with for many years.

Despite their well-known and significant symptom burden, the psychological manifestations of MPNs and their characteristic associations have yet to be adequately described. Rates of psychological distress have been obtained for most oncologic and hematologic malignancies, but none of the large crosssectional studies of distress prevalence have included MPNs. ${ }^{3}$ In general, symptom burden in cancer tends to worsen quality of life (QoL), challenges patients' coping skills, and is associated with distress, anxiety, and depression. ${ }^{14}$ For instance, advanced cancer is almost consistently associated with the psychological consequence of distress/anxiety/depression that is frequently attributed to symptom burden, and not simply knowledge of unfavorable prognosis. ${ }^{15}$ Also, treatments such as interferon, for example, carry a significant association with the development of depression and adverse psychological states, and are routinely used in patients with MPNs. Psychological manifestations of cancer portend worse outcomes in most malignancies but can be treated adequately when recognized in most cases. ${ }^{16}$

Managing the psychiatric and psychological manifestations of cancer is increasingly important for meeting patient care standards. For instance, screening for distress is now mandated by the American Academy of Surgeons' Commission on Cancer to meet cancer center accreditation standards, which should include patients with MPNs. ${ }^{17}$ Although NCCN has endorsed the Distress Thermometer and Problem List (DT\&PL) as an appropriate screening tool, ${ }^{18}$ there is not an accepted gold standard tool for distress screening. A 2-step screening with the DT\&PL and another measure has also been advocated for greater information. ${ }^{19,20}$ The Hospital Anxiety Depression Scale (HADS) is a useful and appropriate tool in patients with chronic illness because it does not use physical symptoms as part of the criteria to screen for anxiety and depression that may otherwise be elevated due to physical illness. ${ }^{21}$

Given the important need to understand the prevalence of distress, anxiety, and depressive symptoms in all cancer subtypes, including MPNs, before instituting distress screening, we have collected baseline distress, anxiety, and depressive symptom data and analyzed their associations with standard disease-specific demographic variables (eg, age, sex, race, length of time with disease) and social/psychological-functioning variables (eg, partnered, working, taking antidepressant medication).

\section{Methods and Materials}

The Mount Sinai Hospital Institutional Review Board approved this study in July 2014. Surveys were collected from participants from May 2015 to October 2015. 
Distress, Anxiety, and Depression in MPNs

\section{Participants}

Men and women with documented MPNs were screened based on inclusion criteria consisting of a confirmed tissue diagnosis of an MPN as identified by the treating physician. Patients selected their disease designation as ET, PV, MF, or other. Exclusion criteria consisted of another cancer diagnosis as identified by the patient. Recruitment occurred over 4 months in a dedicated MPN clinic. New and established patients were recruited to participate in the survey.

\section{Procedure}

Patients were asked to participate by either a clinic receptionist or treating staff (ie, nurse practitioner, hematologist). They were told that the survey was anonymous, was part of a research initiative, and that it did not relate to their ongoing care. Available psychological services were listed in the survey and patients were asked to discuss any concerns with clinic staff and, in particular, to tell a staff member if they felt significantly depressed or had suicidal ideation. A board-certified psychiatrist oversaw the study and was available for consultation. Participants completed surveys while waiting in the clinic office space before or after their appointments and returned them directly to clinic staff.

\section{Measures}

Patients were asked to provide demographic information, including age, sex, marital and working status, disease type (ET, PV, MF, other), length of time with disease $(<1$ year, $1-3$ years, $3-5$ years, $5-10$ years, $>10$ years), and whether they were currently taking an antidepressant medication. In addition, patients completed the DT\&PL and HADS, and were also asked to provide any additional information or individual comments about coping with an MPN and its effect on patients' lives.

The DT\&PL is suggested by NCCN to screen for distress. ${ }^{18}$ It has been used widely by cancer institutions to meet the Commission on Cancer distressscreening mandate for accreditation in 2015.22,23 The DT\&PL is a one-item measure of distress and a Problem List comprising questions relating to Practical, Family, Emotional, Spiritual, and Physical issues. The Distress Thermometer part of the DT\&PL has a range of scores from 0 to 10 , and a cutoff score of 4 or more has been accepted by NCCN as indicating a positive screen for distress. The Problem List part of the DT\&PL is meant to accompany the Distress Thermometer but is not a screening tool; it consists of questions with yes/no response options to help identify key areas of concern.

The HADS is a 14-question psychometric measure that was developed to identify "caseness" (possible and probable cases) of anxiety and depressive disorders among patients in hospital clinics. ${ }^{24}$ Physical symptoms are excluded. The HADS is divided into an anxiety subscale (HADS-A) and a depression subscale (HADS-D). Responses are scored 0 to 3 points, such that each individual HADS (ie, HADS-A and HADS-D) may garner between 0 and 21 points. Multiple cutoffs have been identified, but a cutoff of 11 (total) or 8 (on each subscale) is most commonly used to identify caseness of both depression and anxiety, with a sensitivity and specificity of 0.80 on average. ${ }^{24,25}$

\section{Statistical Analysis}

The primary outcomes of this study were the clinical associations with distress (eg, DT\&PL and HADS), anxiety (eg, HADS-A), and depression (eg, HADSD) among patients with MPNs. Specifically, rates of meeting distress screening, anxiety, and depression criteria were compared by patient characteristics (age, race, sex, disease type, time with disease, married, working, and if taking an antidepressant). Also, prevalence of distress (eg, DT\&PL) and DT\&PL problem list areas (practical/family/emotional/physical), anxiety (eg, HADS-A), and depression (eg, HADS-D), and clinical characteristics (eg, time with disease, working, married) were compared across disease MPN groups (eg, ET, PV, MF, and other). Chisquare tests were used to assess the bivariate associations between preferences and categorical variables. Independent $t$ tests were used to assess the bivariate associations between continuous explanatory and outcome variables. Statistical procedures were performed using SPSS version 22.0 (IBM Corp., Armonk, NY) and statistical tests were 2-tailed with a 5\% significance level.

\section{Results}

Of the 117 participants that completed the survey (78\% response rate), 69 were women $(60.5 \%)$ and 45 were men $(39.5 \%)$. The average age was 57.7 years (SD, 14.8), 65.2\% were married, and $55.8 \%$ 
were working. A total of 109 participants indicated their MPN type: 34 (31.2\%) had PV; 31 (28.4\%) had ET; 31 (28.4\%) had MF; and 13 (11.9\%) had another type of MPN. Most members in the sample were white $(n=86 ; 81.1 \%)$ followed by Latino $(\mathrm{n}=11 ; 10.1 \%)$, black $(\mathrm{n}=8 ; 7.3 \%)$, Asian $(\mathrm{n}=3$; $2.8 \%)$, and other $(n=5 ; 4.6 \%)$. Given the smaller numbers, nonwhite race/ethnicity was aggregated $(n=27 ; 24.8 \%)$. The length of time with disease was more heavily represented by patients with chronic disease as follows: less than 1 year $(n=8 ; 7.5 \%), 1$ to 3 years $(n=21 ; 19.8 \%), 3$ to 5 years $(n=25 ; 23.6 \%)$, 5 to 10 years $(n=21 ; 19.8 \%)$, and more than 10 years $(n=31 ; 29.2 \%)$. There was not a significant difference between chronicity of disease (length of time with disease) and disease type (Table 1). In total, 18 participants (15.4\%) were taking an antidepressant.

The average distress as measured by the DT\&PL was 3.14 (2.83), with $40.4 \%$ meeting NCCN-designated distress screening criteria. HADS screening (ie, for distress) averaged 8.97 (7.44), with 38.5\% meeting the HADS screening criteria of a score of 11 or more. Anxiety-specific screening (eg, HADSA) averaged 5.54 (4.37), with 31.3\% meeting screening criteria (eg, HADS-A $\geq 8$ ). Depressionspecific screening criteria (eg, HADS-D) averaged 3.4 (3.4), with $12.5 \%$ meeting screening criteria (eg, HADS-D $\geq 8$ ).

Correlation strengths between distress (DT\&PL), individual Problem List components (eg, Practical, Family, Emotional, Spiritual, Physical), distress (HADS), anxiety (HADS-A), and depression (HADS-D) were all statistically significant. Pearson coefficient scores greater than 0.6 (ie, approaching colinearity) were noted between anxiety (HADS-A) and distress (HADS) $(P=.833)$; anxiety (HADS-A) and Emotional $(r=.726)$; depression (HADS-D) and Physical $(r=.652)$; distress (HADS) and Emotional $(r=.644)$; Family and Practical $(r=.619)$; and depression (HADS-D) and anxiety (HADS-A) $(r=.608)$.

General distress screening criteria (DT\&PL $\geq 4$, HADS $\geq 11$ ) were more commonly met in nonwhite patients $(P=.045$ and $P=.015$ for DT\&PL $\geq 4$ and HADS $\geq 11$, respectively) and those with either PV or MF but not ET (DT\&PL $\geq 4 ; P=.038$ ) (Table 1 ). Otherwise, there was no association between age, gender, marriage or working status, or length of time with disease and meeting distress/anxiety/depression screening criteria. Patients taking antidepressants were associated with meeting distress screening criteria (DT\&PL $\geq 4)(P=.02)$ but were not associated with meeting anxiety (HADS-A $\geq 8$ ) or depression (HADS-D $\geq 8$ ) screening criteria.

The average distress (ie, DT\&PL) was higher for PV (3.86), MF (3.12), and other MPN (4.33) than it was for ET $(1.81 ; P=.016)$ (Table 2$)$. However, there were no other significant differences by disease group for distress (ie, HADS), anxiety (HADS-A), or depression (HADS-D). There was no association between disease type and marriage or working status or taking antidepressants (Table 2).

Table 3 displays an estimate of symptom burden using the Problem List from the DT\&PL and its relationship to distress, anxiety, or depression outcomes. Multivariate analysis found that Emotional issues (ie, on the DT\&PL Problem List) predicted for elevated distress (DT\&PL), anxiety (HADS-A), and depression (HADS-D), whereas Spiritual and Physical issues predicted for depression (HADS-D). Outcomes were controlled by age.

Patients' comments fit into 2 distinct categories that described either coping or symptom burden. A total of 26 comments were provided by patients and are listed in supplemental eAppendix 1 (available with this article at JNCCN.org). Patients described either feeling well without symptoms, focusing on a positive aspect of their condition, or they expressed the burden of the disease with the multiple losses that they may have encountered as a result of the MPN.

\section{Discussion}

Patients with BCR-ABL-negative MPNs experience clinically relevant levels of distress, anxiety, and depression that require psychological attention. Rates of distress and anxiety were particularly elevated, which is concerning given the relatively lengthy amount of time that patients live with MPNs. Although $\mathrm{QoL}$ and symptom burden experiences are well documented for patients with BCR-ABL-negative MPNs, rates of meeting distress, anxiety, and depression criteria have not yet been documented. This study demonstrates the importance of addressing the psychological manifestations (ie, distress, anxiety, and depression) of BCR-ABL-negative MPNs, which are similar to the rates seen in other advanced malignancies (eg, breast or lung cancer) but have not garnered the same 
Distress, Anxiety, and Depression in MPNs

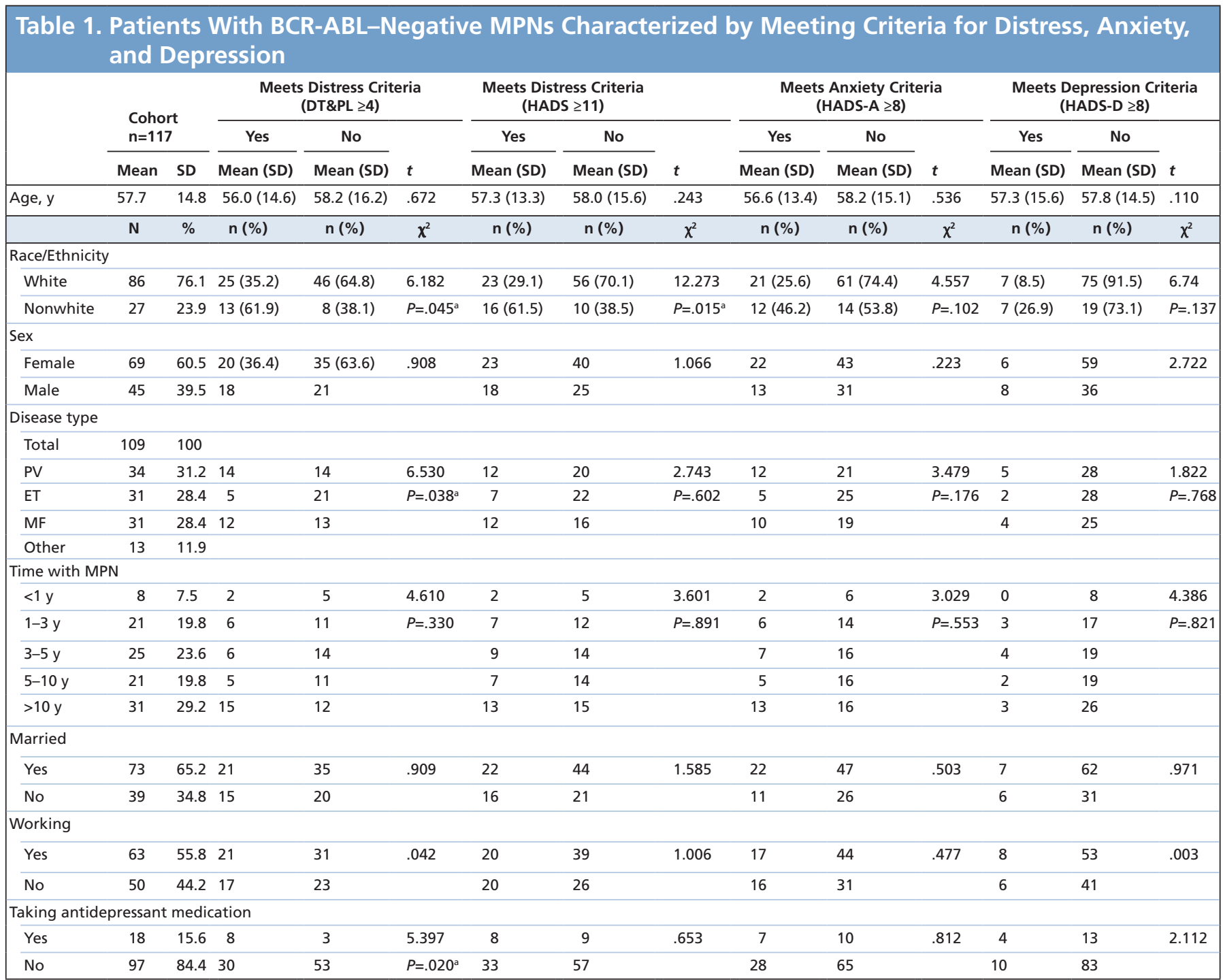

Abbreviations: DT\&PL, Distress Thermometer and Problem List; ET, Essential Thrombocytosis; HADS, Hospital Anxiety Depression Scale; HADS-A, Hospital Anxiety Depression Scale-Anxiety; HADS-D, Hospital Anxiety Depression Scale-Depression; MF, myelofibrosis; MPN, myeloproliferative neoplasm; PV, polycythemia vera. ${ }^{\mathrm{a}} \mathrm{P}<.05$.

amount of diagnostic or therapeutic attention. ${ }^{26,27}$ The NCCN-recommended distress cutoff (DT\&PL 24) had discriminative ability to detect distress variation based on race/ethnicity, disease type (eg, lower distress with ET), or taking an antidepressant (eg, higher distress if taking an antidepressant). This may mean that patients with these chronic disease states harbor continued distress for many years and those at greater risk (eg, already on an antidepressant) might be identified through risk factors.

Symptom burden may play an etiologic role in the development of psychological symptoms (eg, distress, anxiety, depression) in patients with BCRABL-negative MPNs. The MPNs that typically have greater symptom burden (ie, PV and MF) were associated with greater distress levels than those with less symptom burden (ie, ET). Additionally, symptom burden, as indicated by the Problem List variables (ie, from the DT\&PL), found that Emotional, Spiritual, and Physical issues predicted for depression, whereas Emotional issues also predicted for distress and anxiety. This association may also correspond to the unique molecular or inflammatory profiles (eg, cytokines) that are seen in patients with MPNs and are 
McFarland et al

Table 2. Characteristics of Patients Separated by Specific Type of BCR-ABL-Negative MPN

\begin{tabular}{|c|c|c|c|c|c|c|c|c|c|}
\hline & \multicolumn{2}{|c|}{$\begin{array}{c}\mathrm{ET} \\
\mathrm{N}=31\end{array}$} & \multicolumn{2}{|c|}{$\begin{array}{l}\text { Polycythemia Vera } \\
\qquad \mathrm{N}=34\end{array}$} & \multicolumn{2}{|c|}{$\begin{array}{c}\text { Myelofibrosis } \\
\mathrm{N}=31\end{array}$} & \multicolumn{2}{|c|}{$\begin{array}{c}\text { Other MPN } \\
\mathrm{N}=13\end{array}$} & \multirow[b]{2}{*}{$\mathbf{F}$} \\
\hline & Mean & SD & Mean & SD & Mean & SD & Mean & SD & \\
\hline Distress (DT\&PL) & 1.8 & 2.1 & 3.86 & 2.7 & 3.12 & & 4.33 & 3.0 & $3.651^{\text {a }}(P=.016)$ \\
\hline Practical & .61 & .92 & 1.0 & 1.6 & .69 & 1.1 & .92 & 1.3 & .546 \\
\hline Family & .48 & .89 & .75 & 1.1 & .29 & .5 & 1.04 & 1.0 & 2.005 \\
\hline Emotional & .74 & 1.3 & 1.77 & 1.7 & 1.63 & 2.0 & 2.00 & 2.1 & $2.229(P=.09)$ \\
\hline Spiritual & .04 & .2 & & & .05 & .2 & .25 & .5 & 1.601 \\
\hline Physical & 1.46 & 1.7 & 2.89 & 3.8 & 2.81 & 2.8 & 4.23 & 4.9 & $2.318(P=.081)$ \\
\hline Distress (HADS) & 8.97 & 7.5 & 9.91 & 7.8 & 8.93 & 7.7 & 11.71 & 7.9 & 1.721 \\
\hline Anxiety (HADS-A) & 4.66 & 3.6 & 6.33 & 5.0 & 5.03 & 4.3 & 7.23 & 4.0 & 1.531 \\
\hline \multirow[t]{2}{*}{ Depression (HADS-D) } & 2.16 & 3.0 & 3.52 & 3.5 & 3.86 & 3.5 & 4.85 & 3.5 & $2.283(P=.084)$ \\
\hline & $\mathrm{n}$ & $\%$ & $\mathrm{n}$ & $\%$ & $\mathrm{n}$ & $\%$ & $\mathrm{n}$ & $\%$ & $x^{2}$ \\
\hline \multicolumn{10}{|l|}{ Married } \\
\hline Yes & 21 & 70.0 & 20 & 62.5 & 17 & 54.8 & 10 & 76.9 & 5.060 \\
\hline No & 9 & 30.0 & 12 & 37.5 & 14 & 45.2 & 3 & 23.1 & \\
\hline \multicolumn{10}{|l|}{ Working } \\
\hline Yes & 20 & 64.5 & 20 & 60.6 & 13 & 43.3 & 6 & 42.9 & 4.025 \\
\hline No & 11 & 35.5 & 13 & 39.4 & 17 & 56.7 & 8 & 57.1 & \\
\hline \multicolumn{5}{|c|}{ Taking antidepressant medication } & 7 & & & & \\
\hline Yes & 5 & 16.1 & 5 & 14.7 & 7 & 22.6 & 1 & 7.7 & 1.815 \\
\hline \multirow[t]{2}{*}{ No } & 26 & 83.9 & 29 & 85.3 & 24 & 77.4 & 12 & 92.3 & \\
\hline & $n$ & $\%$ & & & & & & & $\chi^{2}$ \\
\hline \multicolumn{10}{|l|}{ Time with MPN } \\
\hline$<1 \mathrm{y}$ & 2 & 6.5 & 2 & 6.1 & 1 & 3.2 & 3 & 25.0 & $20.265(P=.062)$ \\
\hline $1-3 y$ & 9 & 29.0 & 4 & 12.1 & 8 & 26.7 & 0 & 0.0 & \\
\hline $3-5 y$ & 3 & 9.7 & 8 & 24.2 & 9 & 30.0 & 5 & 41.7 & \\
\hline $5-10 y$ & 8 & 25.8 & 6 & 18.2 & 4 & 13.3 & 3 & 25.0 & \\
\hline$>10 y$ & 9 & 29.0 & 13 & 39.4 & 8 & 26.7 & 1 & 8.3 & \\
\hline
\end{tabular}

Abbreviations: DT\&PL, Distress Thermometer and Problem List; ET, Essential Thrombocytosis; HADS, Hospital Anxiety Depression Scale; HADS-A, Hospital Anxiety Depression Scale-Anxiety; HADS-D, Hospital Anxiety Depression Scale-Depression; MPN, myeloproliferative neoplasm. a $P<.05$.

associated with greater symptom burden. ${ }^{28}$ JAK inhibition with drugs such as ruxolitinib reduce symptom burden (eg, fatigue, splenomegaly) and proinflammatory cytokine biomarkers. Psychological effects of JAK inhibition have not been measured previously and deserve investigative attention. Results could be correlated with biological inflammatory markers.

Further, overall distress (eg, meeting distress screening criteria) was higher for nonwhite patients, which is consistent with long-standing health inequities for minority populations faced with a chronic illness. ${ }^{29}$ Stigma and other barriers to mental health care among ethnic minorities may account for this difference. Also, disproportionate rates of symptom burden or communication concordance differences may account for this difference. ${ }^{30}$

Limitations to this study include its exploratory, cross-sectional nature with limited numbers of patients in each group and a lack of specific descrip- tions for the "other" group. Although the scales are validated tools, a gold-standard test or clinical interview to assess distress/anxiety/depression was not performed. Therefore, these results are approximations that are exploratory and require further study before definitive conclusions can be drawn. The associations should be further assessed. Another limitation is the use of aggregated demographic data with a small number of nonwhite participants. Additionally, only 13 participants represented the "Other MPN" group, and data regarding their disease type was not collected. Participants in the "Other MPN" group may have been distressed by not having a standard MPN type or had greater symptom burden (eg, pruritus). Also, a validated QoL tool to assess symptom burden would have enriched the association data and should be considered in a future study design due to the high symptom burden in MPNs. Given that the HADS was originally validated in 
Distress, Anxiety, and Depression in MPNs

\begin{tabular}{|c|c|c|c|c|}
\hline Outcome Variable & $\begin{array}{l}\text { Regression Coefficient } \\
\text { (SE) }\end{array}$ & Beta & $t$ Value & $P$ Value \\
\hline \multicolumn{5}{|l|}{ Distress (DT\&PL) } \\
\hline Practical & $.063(.28)$ & .029 & .226 & .822 \\
\hline Family & $.354(.37)$ & .119 & .986 & .336 \\
\hline Emotional & $.599(.17)$ & .394 & 3.450 & $.001^{\mathrm{a}}$ \\
\hline Spiritual & - & - & - & - \\
\hline Physical & $.197(.09)$ & .244 & 2.265 & $.026^{\mathrm{b}}$ \\
\hline \multicolumn{5}{|c|}{ Adjusted $R^{2}=.363$} \\
\hline \multicolumn{5}{|c|}{ Distress (HADS-Total) } \\
\hline Practical & $-.461(.68)$ & -.074 & -.802 & .425 \\
\hline Family & $.429(.73)$ & .050 & .585 & .560 \\
\hline Emotional & $2.303(.39)$ & .570 & 5.896 & $<.000^{c}$ \\
\hline Spiritual & $3.818(2.22)$ & .129 & 1.721 & .09 \\
\hline Physical & $.809(.23)$ & .288 & 3.477 & $.001^{\mathrm{a}}$ \\
\hline \multicolumn{5}{|c|}{ Adjusted $R^{2}=.671$} \\
\hline \multicolumn{5}{|c|}{ Anxiety (HADS-A) } \\
\hline Practical & $-.040(.37)$ & -.010 & -.100 & .920 \\
\hline Family & $.671(.50)$ & .127 & 1.330 & .188 \\
\hline Emotional & $1.475(.27)$ & .605 & 5.485 & $<.000^{c}$ \\
\hline Spiritual & $.709(1.53)$ & .039 & .464 & .644 \\
\hline Physical & $.271(.16)$ & .157 & 1.693 & .095 \\
\hline \multicolumn{5}{|c|}{ Adjusted $R^{2}=.585$} \\
\hline \multicolumn{5}{|c|}{ Depression (HADS-D) } \\
\hline Practical & $-.427(.28)$ & -.157 & -1.506 & .137 \\
\hline Family & $-.317(.36)$ & -.084 & -.879 & .382 \\
\hline Emotional & $.704(.19)$ & .404 & 3.656 & $<.000^{c}$ \\
\hline Spiritual & $3.078(1.09)$ & .238 & 2.814 & $.006^{\mathrm{a}}$ \\
\hline Physical & $.517(.12)$ & .420 & 4.508 & $<.000^{c}$ \\
\hline
\end{tabular}

Abbreviations: DT\&PL, Distress Thermometer and Problem List; HADS, Hospital Anxiety Depression Scale; HADS-A, Hospital Anxiety Depression Scale-Anxiety; HADS-D, Hospital Anxiety Depression Scale-Depression.

${ }^{a} P<.01$.

${ }^{\mathrm{b}} P<.05$.

c $P<.001$.

primary care patients, the cutoff points for screening with patients with cancer may be too high.

\section{Conclusions}

This study provides cross-sectional prevalence data for distress, anxiety, and depression levels among patients with BCR-ABL-negative MPNs. Psychological data in this area have been lacking historically. These baseline data could be interpreted as baseline rates of distress, anxiety, and depression that may be seen in the context of mandated institutional distress screening. There is a need for greater attention to the psychological symptoms associated with BCRABL-negative MPNs, especially given the length of time with the disease. The potential link between symptom burden (eg, MPN-specific symptoms and complications), inflammatory markers, QoL, and meeting distress/anxiety/depression screening criteria in patients with MPNs should be explored further.

\section{References}

1. Fitzgerald P, Lo C, Li M, et al. The relationship between depression and physical symptom burden in advanced cancer. BMJ Support Palliat Care 2015;5:381-388

2. Breen SJ, Baravelli CM, Schofield PE, et al. Is symptom burden a predictor of anxiety and depression in patients with cancer about to commence chemotherapy? Med J Aust 2009;190(7 Suppl):S99-104.

3. Zabora J, BrintzenhofeSzoc K, Curbow B, et al. The prevalence of psychological distress by cancer site. Psychooncology 2001;10:19-28.

4. Brintzenhofe-Szoc KM, Levin TT, Li Y, et al. Mixed anxiety/depression symptoms in a large cancer cohort: prevalence by cancer type. Psychosomatics 2009;50:383-391.

5. Mesa RA, Niblack J, Wadleigh M, et al. The burden of fatigue and quality of life in myeloproliferative disorders (MPDs): an international Internetbased survey of 1179 MPD patients. Cancer 2007;109:68-76.

6. Scherber R, Dueck AC, Johansson P, et al. The Myeloproliferative Neoplasm Symptom Assessment Form (MPN-SAF): international prospective validation and reliability trial in 402 patients. Blood 2011;118:401-408. 
7. Mondet J, Hussein K, Mossuz P. Circulating cytokine levels as markers of inflammation in Philadelphia negative myeloproliferative neoplasms: diagnostic and prognostic interest. Mediators Inflamm 2015;2015:670580.

8. Raison CL, Capuron L, Miller AH. Cytokines sing the blues: inflammation and the pathogenesis of depression. Trends Immunol 2006;27:24-31.

9. McGregor BA, Antoni MH. Psychological intervention and health outcomes among women treated for breast cancer: a review of stress pathways and biological mediators. Brain Behav Immun 2009;23:159-166.

10. Costanzo ES, Lutgendorf SK, Sood AK, et al. Psychosocial factors and interleukin-6 among women with advanced ovarian cancer. Cancer 2005; 104:305-313.

11. Irwin MR, Miller AH. Depressive disorders and immunity: 20 years of progress and discovery. Brain Behav Immun 2007;21:374-383.

12. Dameshek W. Some speculations on the myeloproliferative syndromes. Blood 1951;6:372-375

13. Tefferi A, Pardanani A. Myeloproliferative neoplasms: a contemporary review. JAMA Oncol 2015;1:97-105.

14. Cella D, Nowinski CJ, Frankfurt O. The impact of symptom burden on patient quality of life in chronic myeloid leukemia. Oncology 2014;87:133147.

15. Mitchell AJ, Chan M, Bhatti H, et al. Prevalence of depression, anxiety, and adjustment disorder in oncological, haematological, and palliativecare settings: a meta-analysis of 94 interview-based studies. Lancet Oncol 2011;12:160-174.

16. Andersen BL, DeRubeis RJ, Berman BS, et al. Screening, assessment, and care of anxiety and depressive symptoms in adults with cancer: an American Society of Clinical Oncology guideline adaptation. J Clin Oncol 2014;32:1605-1619.

17. Wagner LI, Spiegel D, Pearman T. Using the science of psychosocial care to implement the new american college of surgeons commission on cancer distress screening standard. J Natl Compr Canc Netw 2013;11:214-221.

18. Holland JC, Jacobsen PB, Andersen B, et al. NCCN Clinical Practice Guidelines in Oncology: Distress Management. Version 2.2016. Accessed December 22, 2015. To view the most recent version of these guidelines, visit NCCN.org.
19. Harter M, Woll S, Wunsch A, et al. Screening for mental disorders in cancer, cardiovascular and musculoskeletal diseases. Comparison of HADS and GHQ-12. Soc Psychiatry Psychiatr Epidemiol 2006;41:56-62.

20. Wang GL, Hsu SH, Feng AC, et al. The HADS and the DT for screening psychosocial distress of cancer patients in Taiwan. Psychooncology 2011;20:639-646.

21. Vodermaier A, Linden W, Siu C. Screening for emotional distress in cancer patients: a systematic review of assessment instruments. J Natl Cancer Inst 2009;101:1464-1488.

22. Bower JE, Bak K, Berger A, et al. Screening, assessment, and management of fatigue in adult survivors of cancer: an American Society of Clinical Oncology clinical practice guideline adaptation. J Clin Oncol 2014;32:1840-1850.

23. Pirl WF, Fann JR, Greer JA, et al. Recommendations for the implementation of distress screening programs in cancer centers: report from the American Psychosocial Oncology Society (APOS), Association of Oncology Social Work (AOSW), and Oncology Nursing Society (ONS) joint task force. Cancer 2014;120:2946-2954.

24. Bjelland I, Dahl AA, Haug TT, Neckelmann D. The validity of the Hospital Anxiety and Depression Scale. An updated literature review. J Psychosom Res 2002;52:69-77.

25. Zigmond AS, Snaith RP. The hospital anxiety and depression scale. Acta Psychiatr Scand 1983;67:361-370.

26. Walker J, Holm Hansen C, Martin P, et al. Prevalence of depression in adults with cancer: a systematic review. Ann Oncol 2013;24:895-900.

27. Massie MJ. Prevalence of depression in patients with cancer. J Natl Cancer Inst Monogr 2004:57-71.

28. Geyer HL, Dueck AC, Scherber RM, Mesa RA. Impact of inflammation on myeloproliferative neoplasm symptom development. Mediators Inflamm 2015;2015:284706.

29. Cooper LA, Powe NR. Disparities in patient experiences, health care processes, and outcomes: the role of patient-provider racial, ethnic, and language concordance. New York, NY: The Commonwealth Fund; 2004.

30. Cooper LA, Roter DL, Johnson RL, et al. Patient-centered communication, ratings of care, and concordance of patient and physician race. Ann Intern Med 2003;139:907-915.

\section{See JNCCN.org for supplemental online content.}

